\title{
Wann sind Sorgen pathologisch?
}

\author{
Jürgen Hoyer Sabrina Heidrich \\ Klinische Psychologie und Psychotherapie, Technische Universität Dresden, Deutschland
}

\section{Schlüsselwörter}

Sorgen - Generalisierte Angststörung · Sorgenexposition . Funktionale Bedingungsanalyse · Emotionsvermeidung · Acceptance and Commitment Therapy

\section{Zusammenfassung}

Pathologische Sorgen sind ungenau definiert. Für die Behandlungsplanung bleiben wichtige Fragen offen: Welche Merkmale sind für die Unterscheidung zwischen behandlungsbedürftigen und nicht behandlungsbedürftigen Sorgen relevant? Welche Art von Sorgen muss wie behandelt werden? Und: Welche Art von Sorgen gilt es eher zu akzeptieren? Wir machen praxisnahe Vorschläge dafür, wie Sorgen mittels einer einfachen Heuristik auch vom Patienten selbst als "pathologisch" identifiziert werden können. Im Sinne eines therapeutischen Arbeitsmodells ergeben sich differentielle Bearbeitungsstrategien, je nachdem, ob es sich um wichtige oder weniger wichtige, auf lösbare oder unlösbare Probleme bezogene sowie angemessene oder überzogene Sorgen handelt. Das vorgestellte Arbeitsblatt zu den Sorgen soll vor allem die wahrgenommene Kontrolle des Patienten stärken und die Psychoedukation zur Generalisierten Angststörung erleichtern.

\section{Alltagssorgen und pathologische Sorgen}

Sorgen sind ein alltägliches Phänomen, das jeder kennt [vgl. Hoyer und Beesdo, 2006]. Sorgen sind gleichzeitig das klinische Leitsymptom der Generalisierten Angststörung, einer häufigen und beeinträchtigenden psychischen Störung [Stiglmayr et al., 2006; Wittchen et al., 2002]. Aber auch Men-

\section{Key Words}

Worry - Generalised anxiety disorder - Worry exposure . Functional analysis - Experiential avoidance .

Acceptance and Commitment Therapy

\section{Summary}

When Are Worries Pathological?

Pathological worries have not yet been clearly defined. As a consequence, practically relevant questions remain open: Which characteristics distinguish worries relevant for treatment from those which are not? What kind of worries has to be treated in which way? And: What kind of worries is rather to be accepted? We propose a simple rationale which helps the therapist and the patient to identify pathological worries. According to this working model, different treatment strategies result depending on whether worries are central or not, whether they relate to a problem which can be solved or not, and whether they seem proportionate or exaggerated. The presented worksheet is meant to strengthen the perceived control of the patient and to help facilitate psychoeducation for generalised anxiety disorder.

schen ohne Generalisierte Angststörung können sich in einem pathologischen Ausmaß Sorgen machen [Ruscio und Borkovec, 2003], und exzessive Sorgen sind auch bei Patienten mit anderen psychischen Störungen - wie der Depression - häufig [Hoyer et al., 2002]. Sorgen scheinen damit auf den ersten Blick ein unklares und wenig spezifisches diagnostisches Kriterium zu sein.

\section{KARGER}

Fax +497614520714

Information@Karger.de

www.karger.com (c) 2009 S. Karger GmbH, Freiburg

Accessible online at:

www.karger.com/ver
Prof. Dr. Jürgen Hoyer

Klinische Psychologie und Psychotherapie

Technische Universität Dresden

Hohe Str. 53, 01187 Dresden, Deutschland

Tel. +49 351 4633-6986, Fax -6955

hoyer@psychologie.tu-dresden.de 
Die bekannteste Definition von Sorgen stammt von Borkovec et al. [1983, 10]. Danach ist das Sich-Sorgen-Machen (worrying) «a chain of thoughts and images, negatively affectladen, and relatively uncontrollable; it represents an attempt to engage in mental problem-solving on an issue whose outcome is uncertain but contains the possibility of one or more negative outcomes; consequently, worry relates closely to the fear process». Sich zu sorgen bedeutet damit eher eine Reihe von wechselnden Gedanken und Vorstellungen (gegenüber einem einzelnen Gedanken); es bedeutet, dass negative Gefühle den Prozess begleiten und dass dies als unkontrollierbar erlebt wird. Zugleich wird das Sich-Sorgen als ein Versuch gekennzeichnet, zukünftige (negative) Ausgänge gedanklich vorwegzunehmen (für zahlreiche weitere Definitionsversuche vgl. Davey und Wells [2006]). Grübeln, wenngleich ein sehr ähnlicher Prozess [Watkins, 2008], bezieht sich hingegen eher auf zurückliegende Ereignisse [Becker und Margraf, 2007].

Obwohl diese exemplarische Definition zugleich den Startpunkt der extensiven klinischen Forschung zu den Sorgen (worries) markiert, so zieht sie keine klare Grenze zwischen Alltagssorgen und pathologischen Sorgen. Diese Grenze auf der Grundlage wissenschaftlicher Daten oder Modelle empirisch genauer zu bestimmen, erweist sich nach wie vor als schwierig. Ruscio et al. [2001] konnten zeigen, dass sich ein Kontinuum der Sorgen ergibt, wenn psychisch unterschiedlich stark gestörte Gruppen untersucht werden. Sorgen sind bei Patientengruppen zwar häufiger und intensiver, aber weder thematisch [Becker et al., 2003] noch qualitativ kategorial unterscheidbar. Dies sollte allerdings nicht zu dem Fehlschluss verleiten, die Störung sei wenig beeinträchtigend [vgl. Hoyer et al., 2003]: Sorgen von Patienten mit Generalisierter Angststörung werden als deutlich weniger kontrollierbar und emotional stärker beeinträchtigend erlebt und sind von stärkeren körperlichen Symptomen begleitet als diejenigen von Gesunden oder von Patienten mit einer anderen Angststörung (Soziale Phobie, Hoyer et al. [2001]). Der Zeitaufwand für diese Sorgen ist in der genannten Studie um den Faktor 10 höher. Dennoch, auch bei diesen Befunden handelt es sich zwar um deutliche, jedoch graduelle und nicht kategoriale Unterschiede. Diagnostisch leicht erkennbare Indikatoren pathologischer Sorgen lassen sich daraus nicht ableiten. Ferner ist selbst von etablierten psychometrischen Instrumenten, wie dem Penn State Worry Questionnaire (Stöber, 1995; abgedruckt in Becker und Margraf [2007]), nicht mehr als ein erstes Datum zur Häufigkeit der klinisch relevanten Sorgenproblematik zu erwarten; zwischen klinischen und alltäglichen Sorgen vermag das Verfahren nicht befriedigend zu trennen [Ruscio et al., 2001; Verkuil et al., 2007].

Das Diagnostische und Statistische Manual Psychischer Störungen [DSM-IV-Textrevision; deutsch: Saß et al., 2003] versucht deshalb, die pathologischen Sorgen näher zu bestimmen. So wird zur Generalisierten Angststörung angeführt, Intensität, Dauer oder Häufigkeit der Angst und Sorge seien «deutlich übertrieben im Vergleich zu der tatsächlichen
Wahrscheinlichkeit oder Auswirkung des gefürchteten Ereignisses». Ferner habe die Person «Schwierigkeiten, die Sorgen zu stoppen». Das zusätzliche, relativ strenge Zeitkriterium von 6 Monaten soll sicherstellen, dass nicht schon Alltagssorgen und -probleme zur Vergabe der Diagnose einer Generalisierten Angststörung führen.

Im Rahmen der Verhaltenstherapie der Generalisierten Angststörung sollten zusätzlich aber die funktionalen Merkmale der Sorgen beachtet werden (vgl. auch den Unterschied zwischen «der Sorge» und «dem Akt des Sich-Sorgens» [Becker und Hoyer, 2005; Gerlach et al., 2008]). Im derzeit wichtigsten Modell der Entstehung und Aufrechterhaltung pathologischer Sorgen, der Vermeidungstheorie der Sorgen von Borkovec [z.B. Borkovec et al., 1998, 2004; Sibrava und Borkovec, 2006] sowie in neueren Ansätzen aus der akzeptanzbasierten Verhaltenstherapie [Mennin et al., 2002; Roemer et al., 2005] wird die dysfunktionale Bedeutung der Sorgen vor allem darin gesehen, dass sie den Zugang zu einer emotionalen Verarbeitung von Befürchtungen erschweren (Emotionsvermeidung).

Grundlagenbefunde zeigen, dass Patienten mit Generalisierter Angststörung verstärkt auf die Wahrnehmung von potentiellen Gefahren ausgerichtet sind [Becker et al., 2001]; zugleich neigen sie zu einer genaueren Wahrnehmung des phasischen Arousals und zu katastrophisierenden Überinterpretationen [Andor et al., 2008]. Wenn sie aber tatsächlich mit bedrohlichen Reizen konfrontiert werden, reagieren sie mit einem einseitig kognitiven Verarbeitungsstil [Borkovec und Inz, 1990]. Idealtypisch scheinen die Patienten der Regel zu folgen: «Wenn ich mich mit dem Thema (der Hauptsorge) intensiv beschäftige, werde ich das nicht aushalten können / werde ich verrückt» usw. Dieser Befürchtung entsprechend findet keine vertiefte emotionale Verarbeitung bedrohlicher Informationen statt [vgl. Grawe, 2004, 165ff]. Insbesondere Borkovec [Borkovec et al., 1998; Sibrava und Borkovec, 2006] hat in seinen Arbeiten darauf hingewiesen, dass den Sorgen selbst, als vorwiegend verbal-abstrakter Aktivität, eine (emotions-)vermeidende Funktion zukommt. Zwei Varianten der Vermeidung spielen eine Rolle [Borkovec et al., 1998]:

Eine «abergläubische» Variante, bei der Patienten meinen, die Auftretenswahrscheinlichkeit eines befürchteten Ereignisses zu senken, wenn sie sich vorher darüber «Gedanken» gemacht haben. Sie fühlen sich dadurch besser vorbereitet. Dies vermittelt ein Gefühl von Sicherheit und Kontrolle. Da es sich bei den befürchteten Katastrophen aber um unwahrscheinliche Ereignisse handelt, wird der Betroffene durch das Nichteintreten in seinem Glauben, dass das Sich-Sorgen hilfreich war, bestärkt.

Im zweiten, wichtigeren Prozess werden Sorgen bewusst oder automatisch eingesetzt, um den mit intensiven Emotionen verbundenen, körperlich erlebten Stress zu reduzieren [Borkovec et al., 1998; Mennin et al., 2002]. Da Sorgen nicht mit erhöhter sympathischer Aktivität einhergehen, sondern das Aufkommen starker emotionaler und körperlicher Symp- 
tome begrenzen, können sie als kurzfristiger negativer Verstärker (Wegfall unangenehmer Spannung) wirksam werden.

Die zuletzt beschriebene subtile Form von Vermeidung ist bei Patienten mit Generalisierter Angststörung typisch [Borkovec und $\mathrm{Hu}, 1990$ ]. Sie vermeiden unangenehme internale Erfahrungen (experiential avoidance), indem sie sich über künftige Ereignisse sorgen. Die durch die Vermeidung fehlende Möglichkeit zur emotionalen Verarbeitung und der Mangel an korrektiven Erfahrungen bewirkt, dass die bedrohlichen Themen (Sorgen) bedrohlich bleiben. Zusätzliche Belastungen und damit zusätzliche Trigger für Sorgen können aber jederzeit hinzukommen. Die Tatsache, dass auf diese Weise die Sorgen offensichtlich immer häufiger werden, führt dann in der Folge zu Gegenregulationsversuchen und Kontrollstrategien.

Sobald Menschen versuchen, ihre Sorgen-Gedanken (im weitesten Sinne) zu kontrollieren, ist der Sorgenprozess symptomatisch bzw. klinisch bedeutsam geworden. Die angestrebte Kontrolle der eigenen Gedanken ist ein nicht zu realisierendes Ziel und führt indirekt zu einer anhaltenden Beschäftigung mit ihnen. Im therapeutischen Setting gilt es, die Patienten zur Einsicht zu geleiten, dass Kontrolle nicht die Lösung ist («control is the problem») [Hayes et al., 1999]. Ein probeweises Aufgeben der Kontrolle bzw. eine aktive Intensivierung der mit den Sorgen verbundenen Vorstellungsinhalte, nämlich die Sorgenexposition [Becker und Margraf, 2007; Hoyer und Becker, 2005], ist geeignet, die zugrundeliegenden Befürchtungen zu falsifizieren: zum Beispiel, die Sorgen machten einen selbst verrückt, seien nicht auszuhalten oder gewännen gänzlich die Kontrolle über den Alltag, wenn nicht gegengesteuert werde. Die Sorgenexposition ist also im Kern eine Befürchtungskonfrontation. Sie zeigt dem Patienten: Kontrolle im Sinne einer Gedankenunterdrückung funktioniert nicht, Gedanken sind aber auch nicht in der Weise unkontrollierbar, dass man ernsthaft Schaden nehmen kann.

Eine weitere und zugleich theoretisch klare Abgrenzung zwischen pathologischen und nichtpathologischen Sorgen, die dem bis hierher entwickelten Verständnis ähnlich ist, wird in dem Ansatz von Wells [1997, 1999] formuliert. In seinem Modell sind alle Sorgen per se zunächst nicht pathologisch. Nicht nur offenes Verhalten, sondern auch kognitives Verhalten selbst werde durch Kognitionen, in letzterem Fall Meta-Kognitionen, gesteuert. Meta-Kognitionen über Sorgen können positiv sein ( «Sorgen helfen mir, mich vorzubereiten»= positive Meta-Kognition) oder negativ («Die Sorgen machen mich eines Tages noch verrückt!» = negative Meta-Kognition). Positive oder negative Meta-Kognitionen, die die Aufrechterhaltung des Sorgenprozesses fördern, sollten in der kognitiven Therapie der Generalisierten Angststörung nach Wells [1997, 1999] im Zentrum stehen. Sorgenexposition sei ein geeigneter Weg, insbesondere die negativen Meta-Kognitionen auf ihren Realitätsgehalt hin zu überprüfen. Negative MetaKognitionen seien für die Störungsaufrechterhaltung aber von besonderer Bedeutung, weil sie «Sorgen über Sorgen» (meta- worries) fördern und weil sie dysfunktionale und kontraproduktive Gegenregulationsversuche initiieren. Kontrollversuchen wie Gedankenunterdrückung, Ablenkung, Vermeidung und Sorgenketten ist gemeinsam, dass sie allenfalls kurzfristig die Häufigkeit von Sorgen mindern können, längerfristig aber das Problem aufrechterhalten. Die Annahme, Sorgen seien gefährlich und nicht kontrollierbar (= negative Meta-Kognition), wird durch diese Gegenregulationsversuche auf paradoxe Weise letztlich gestützt. Da es sich bei diesen sogenannten negativen Meta-Kognitionen über die Sorgen um ein relativ komplexes Konstrukt handelt, ist ihre Exploration in der Therapie nicht einfach und bedarf des speziellen Trainings (praktische Hinweise hierzu bei Wells [1997]). Unserer klinischen Beobachtung nach lassen sich negative Meta-Kognitionen insbesondere im Kontext der kognitiven Vorbereitung zur Sorgenexposition gut identifizieren. Fragt man Patienten, was passieren könnte, wenn sie sich in der Vorstellung intensiv mit ihrer Hauptsorge beschäftigen, so antworten sie nicht selten: «Ich könnte verrückt werden» (oder Ähnliches). Gerade für ein Überprüfen solcher (meta-kognitiver) Befürchtungen ist die Sorgenexposition indiziert.

Für Patienten haben die Sorgen häufig eine emotionsvermeidende Funktion; dies führt aber dazu, dass sich gerade dann, wenn viele Emotionen im Spiel sind, also in Krisen- und Belastungszeiten, die Häufigkeit und Intensität der Sorgen stark ausweitet. Wenn die Patienten dann versuchen gegenzuregulieren, werden die Sorgen eher stärker, und dies wiederum scheint bestehende negative Meta-Kognitionen (z.B. «Ich werde meiner Sorgen nicht mehr Herr») zu bestätigen.

Diese Zusammenfassung vereinfacht die Befunde der Forschung zu pathologischen Sorgen und zur Generalisierten Angststörung. Ein solches, einfaches Störungsmodell ist für die kognitive Verhaltenstherapie der Generalisierten Angststörung unerlässlich [Helbig und Hoyer, 2007]. Die Ableitung eines solchen Modells ist die Voraussetzung dafür, Patienten zur Sorgenexposition zu motivieren [Becker und Margraf, 2007; Hoyer und Becker, 2005]. Sorgenexposition bedeutet dabei, Sorgenszenarien zu entwickeln, die konkret und detailgenau sind und die es deshalb erlauben, die mit ihnen verbundenen Befürchtungen in der Vorstellung zu erleben und emotional zu verarbeiten [Hoyer und Becker, 2005].

\section{Bedingungsanalytisches Verständnis der Sorgen}

Für die Vorbereitung der Sorgenexposition ist die Auswahl «relevanter» Sorgenszenarien zentral. Welche Szenarien bzw. welche Sorgen sind geeignet? Auch bei Patienten mit einer Generalisierten Angststörung sind nicht alle Sorgen dysfunktional. Die Themen der Sorgen sind die gleichen wie bei Gesunden [Becker et al., 2003]. Sorgen haben möglicherweise auch eine positive Funktion (s.u.), wenngleich dies in der Literatur umstritten ist [vgl. Davey, 2006]. Für die Behandlungsplanung gilt: Patienten, die das Ziel verfolgten, sich niemals 
irgendwelche Sorgen zu machen, wären nicht erfolgreich zu behandeln [Eifert und Forsyth, 2005], und schon allein aus diesem Grund ist es sinnvoll, «harmlose» (oder angemessene) von klinisch relevanten Sorgen zu unterscheiden.

Als Gradmesser für diese Unterscheidung schlagen wir in dieser Arbeit eine bedingungsanalytische Diagnostik der Sorgen vor. Dabei hat es sich in der klinischen Arbeit bewährt, zwischen wichtigen (zentralen und wiederkehrenden) und weniger wichtigen Sorgen und Sorgenbereichen zu unterscheiden, Sorgen über lösbare von solchen über unlösbare Probleme zu trennen und Sorgen, die angesichts massiver akuter Bedrohungen entstehen, anders $\mathrm{zu}$ werten als überzogene Sorgen über unwahrscheinliche (wenn auch gravierende) Ereignisse.

Weniger wichtige Sorgen. Die Generalisierte Angststörung ist unter anderem dadurch definiert, dass die Sorgen nicht nur auf einzelne Bereiche beschränkt sind. Subjektiv erleben Patienten es oft so, dass sie vor Sorgen nicht mehr ein noch aus wissen, und es kommt ihnen so vor, als machten sie sich über «alles Mögliche» Sorgen. Eine genaue Exploration der Sorgenthemen und Sorgenzeiten mittels strukturierter Tagebücher ist an dieser Stelle unerlässlich. Dabei lassen sich in der Regel sogenannte Sorgenketten [Becker und Hoyer, 2005; Becker und Margraf, 2007] identifizieren. Dies können eher «katastrophisierende Ketten» (innerhalb eines Themenbereiches) oder thematische Sprünge sein. Die Funktion letzterer, also der oft automatisierten Verkettung «kleinerer» Sorgenbelange, ist dabei eher, von den emotional bedeutsameren Themen abzulenken: Es wird von Sorge zu Sorge gesprungen und es wäre therapeutisch ungünstig, diese Sorgen jeweils inhaltlich aufzugreifen, da ihnen im Wesentlichen eine Ablenkungsbzw. Vermeidungsfunktion zukommt $\left(\mathrm{C}^{-}\right.$gestrichen; die mit der Hauptsorge verbundenen ängstlichen Vorstellungen und die damit verbundene Anspannung werden reduziert). Stattdessen ist es wichtig, den Patienten auf die Hauptsorge und die in diesem Zusammenhang vermiedenen Themen zurückzuführen. Die Unterscheidung zwischen «wichtigen» und «weniger wichtigen» Sorgen sollte der Patient aber möglichst frühzeitig selbst vornehmen. Unserer klinischen Erfahrung nach gelingt dies Patienten in Verbindung mit der gezielt eingesetzten Psychoedukation schnell. Unterstützend können gegebenenfalls kognitive Techniken wie die 100-Jahres-Regel («Wird das für Sie in 100 Jahren noch wichtig sein?»), die graphische Einordnung der jeweiligen Sorgen auf einem Kontinuum möglicher «Katastrophen» oder die Frage «Wie viele Sorgen ist dieses Problem wert?» eingesetzt werden [vgl. Butler und Hope, 2007]. Allein dieses «Aussortieren» von Sorgen kann bereits am Anfang der Therapie zu einer bedeutsamen Entlastung führen, weil deutlich wird, dass die Sorgen nicht so verzweigt und endlos sind, wie es subjektiv anfangs gewirkt hat. Auch wenn den «weniger wichtigen», oft in sogenannten Sorgenketten auftretenden Sorgen auf diese Weise therapeutisch relativ leicht begegnet werden kann, so haben sie andererseits eine emotionsregulierende und -vermeidende Funkti- on, die es im Rahmen der Psychoedukation (bzw. des Modells der Aufrechterhaltung) zu berücksichtigen gilt.

Sorgen in Zusammenhang mit lösbaren Problemen. Sorgen sind nicht immer dysfunktional. Grundsätzlich sind Sorgen auch ein Versuch, zukünftige Katastrophen zu verhindern [Davey et al., 1996; Davey, 2006] oder Was-wäre-wenn?-Fragen zu generieren, um Unsicherheit zu reduzieren [Dugas et al., 1997]. Sie verweisen damit auch auf Probleme, die lösbar sind: Zwar kann die Funktionalität immer nur in Abhängigkeit vom Kontext (und damit im Gesamtzusammenhang der funktionalen Bedingungsanalyse) beurteilt werden, aber wenn Sorgen etwa zum Abschluss einer Reiserücktrittsversicherung führen, die intensivere Vorbereitung auf eine Prüfung motivieren oder der Prüfung der Bremsen eines Autos vorausgehen, um nur einige Beispiele zu nennen, so kann dies funktional, und damit für die Behandlung wenig relevant, sein. Solche Sorgen sollten nicht für die Sorgenexposition ausgewählt werden, zumindest nicht solange sie nicht zu problematischen Reaktionen führen. Wenn eigentlich lösbare Probleme zu fortgesetzten Sorgen führen, kann stattdessen ein systematisches Problemlösetraining indiziert sein [Hoyer et al., 2007], wobei unbedingt zu berücksichtigen ist, dass die für Personen mit chronischen Sorgen typische negative Stimmung nicht als Kriterium für das weitere Generieren von neuen Sorgen herangezogen wird [Davey, 2006]. Auch Sorgen über lösbare Probleme können Teil der Aufrechterhaltung der Störung sein, weil die Patienten zwar häufig über mögliche Problemlösungen nachdenken, den entscheidenden Schritt ins Handeln aber nicht machen. Dies ist mit einem Problemlösetraining gut bearbeitbar.

Sorgen im Zusammenhang mit akuten Belastungen. Sorgen können nach unserer klinischen Beobachtung unkontrollierbar sein und auch der Funktion der Emotionsregulation (und -vermeidung) dienen, ohne dass sie zu den typischen pathologischen Sorgen zu zählen sind: Dies ist der Fall, wenn akute und schwerwiegende Belastungen vorliegen (zum Beispiel: beim Partner sind Anzeichen für eine schwerwiegende Erkrankung sichtbar). In diesen Fällen geht es zwar möglicherweise auch um Emotionsvermeidung, dies muss aber nicht Ausdruck des typischen kognitiven Stils der Person sein, sondern kann auch Ausdruck der existenziellen Ausnahmesituation sein. Auch in diesem Fall muss die Person möglicherweise lernen, Emotionen zuzulassen (zum Beispiel die Angst, der Partner könnte sterben). Bei einer solchen akuten Situation könnte eine Exposition in sensu eine Überforderung darstellen, sofern dieses Vorgehen nicht bereits in der Therapie etabliert wurde.

Alle diese Sorgen können bei einer einzelnen Person zusätzlich zu den für Patienten mit Generalisierter Angststörung zentralen Sorgen gegeben sein. Die Arbeit mit den zentralen Sorgen wird nicht selten erschwert, indem die Patienten immer wieder neue Sorgen vorbringen, deren Funktion im Wesentlichen ist, die Beschäftigung mit den am meisten gefürchteten Themen zu vermeiden. 
Abb. 1. Arbeitsblatt für Patienten [mod. nach Hoyer et al., 2007].

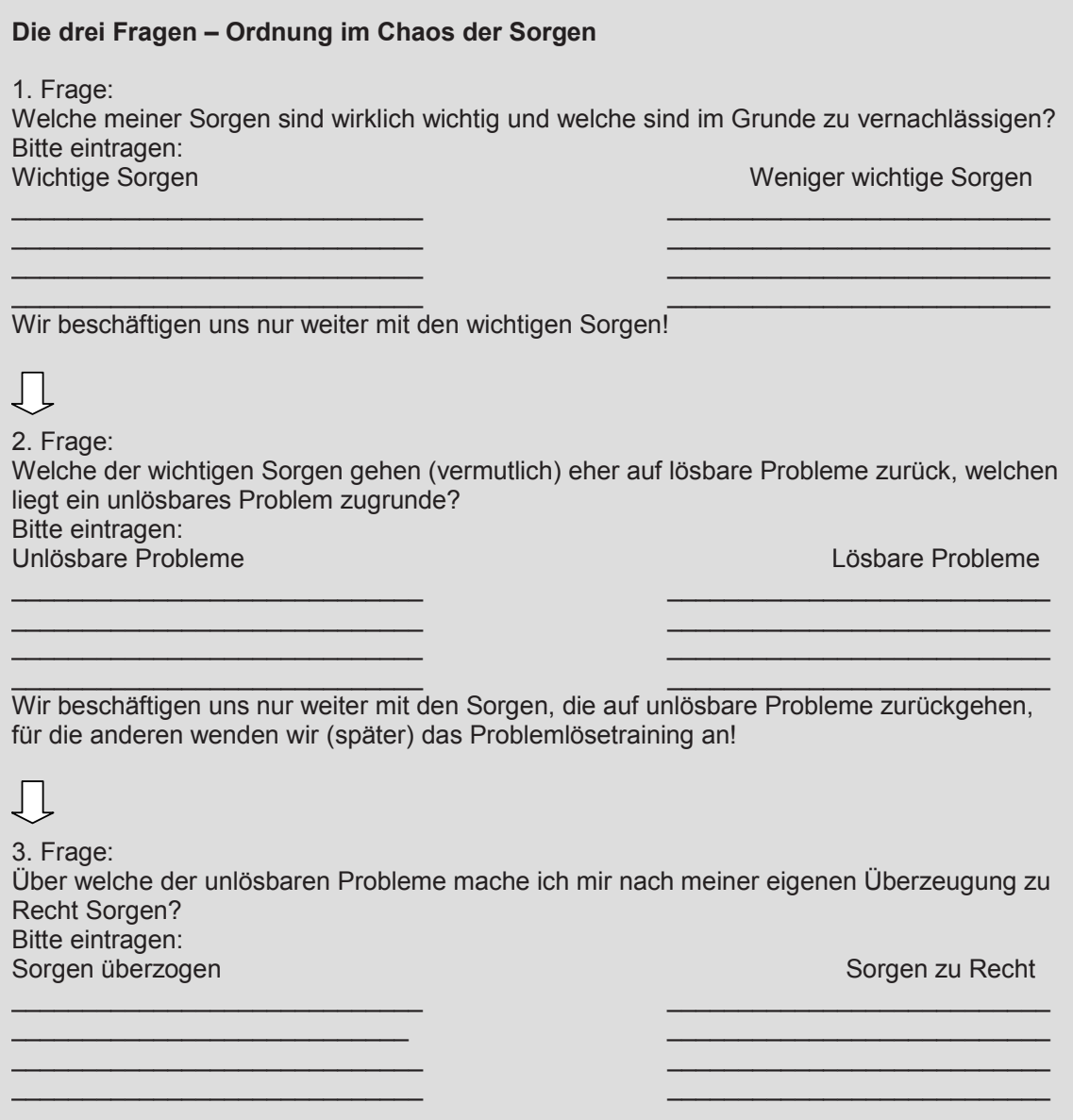

\section{Einfache Psychoedukation zu den Sorgen}

Das von uns vorgeschlagene Ordnungsprinzip zur Unterscheidung der Funktionen von Sorgen gilt es, auf möglichst klare und ökonomische Weise den Patienten zu vermitteln. Wir haben dafür ein einfaches Arbeitsblatt entwickelt, das weitgehend selbsterklärend ist (Erklärung für Patienten auch in Hoyer et al. [2007]) und das das bei Becker und Hoyer [2005] sowie Becker und Margraf [2007] beschriebene Vorgehen unterstützt (Abb. 1).

Allein das Bearbeiten der Taxonomie der Sorgen geht für viele Patienten mit großer Entlastung einher. Durch das taxonomische System und die entsprechende Psychoedukation lernen die Patienten, in ihrem zunächst als chaotisch empfundenen System der Sorgen Ordnung herzustellen. Patienten haben uns berichtet, die Sorgen seien insgesamt für sie weniger bedrohlich, seitdem sie sie entsprechend einordnen können. Gleichzeitig hilft die hier vorgestellte Heuristik aber auch Therapeuten, die richtigen Sorgen für die Sorgenexposition auszuwählen. Unsicherheiten bei diesem Auswahlprozess könnten auch zur Zurückhaltung beim Einsatz geeigneter Expositionsmethoden beitragen [vgl. Böhm et al. 2008]. Daher regen wir an, hinsichtlich der vom Patienten erlebten Sorgen bereits in den ersten Stunden der Behandlung der Genera- lisierten Angststörung eine klare Struktur herzustellen. Es bietet sich an, frühzeitig Übungsblätter, auf denen die Patienten beschreiben müssen, wie ihre Sorgen auf den o.g. drei Dimensionen einzuordnen sind, zu vermitteln. Dieser Ansatz ist ohne Weiteres kompatibel mit dem Manual zur Sorgenexposition von Becker und Margraf [2007]. Dabei gilt allerdings der Hinweis, dass differentialdiagnostisch mittlere und schwere depressive Episoden ausgeschlossen oder bereits remittiert sein sollten. Sowohl die Selbstselektion geeigneter Sorgen als auch die Sorgenexposition selbst dürften bei akuter depressiver Symptomatik eine Überforderung darstellen [Becker und Hoyer, 2005; Becker und Margraf, 2007].

\section{Auf die Sorgen fokussiertes Vorgehen}

Das hier vorgestellte Vorgehen ist ein Baustein einer Therapiestrategie (siehe Fallbeispiel), die den Fokus vor allem auf die Sorgen legt. Die meisten empirisch überprüften verhaltenstherapeutischen Programme zur Behandlung der Generalisierten Angststörung sind demgegenüber Beispiele der multimodalen Verhaltenstherapien [vgl. Bär et al., 2007]. Die Bearbeitung pathologischer Sorgen mit der Sorgenexposition ist in diesen Fällen nur ein Behandlungselement unter vielen. 
Fallbeispiel

Die 56-jährige Patientin stellte sich mit «anhaltenden Angstgefühlen», Nervosität, innerer Unruhe und Anspannung, Erschöpfbarkeit, Ein- und Durchschlafstörungen sowie Konzentrationsstörungen vor. Sie mache sich «pausenlos Sorgen» über verschiedene Bereiche ihres Lebens, wie Familie, Arbeit, ihre Gesundheit sowie nahestehender Personen, Finanzen und alltägliche Probleme. Die Sorgen wüchsen ihr über den Kopf, sie könne aber nichts dagegen tun.

Die ersten therapeutischen Schritte umfassten die Informationsvermittlung zur Diagnose der Generalisierten Angststörung, die Entwicklung eines individuellen Störungsmodells, die gemeinsame Erarbeitung der Bedingungsanalyse sowie die Anleitung der Patientin zur systematischen Selbstbeobachtung ihrer Sorgen- und Anspannungsepisoden. Durch die nachfolgende Anwendung des Ordnungsschemas für die Sorgen erlebte die Patientin binnen kurzer Zeit eine deutliche Reduktion der Häufigkeit und Frequenz von Sorgenepisoden. Zugleich konnte sie anders mit ihren Sorgen umgehen: Die als weniger wichtig beurteilten Sorgen (Erledigung von Routinetätigkeiten; anstehende Reparaturen; Klärung von Meinungsverschiedenheiten mit Freunden) verloren an Bedrohlichkeit. Wichtige lösbare Probleme (Zeitmanagement bei der Arbeit; Organisation der Enkelkindbetreuung) konnten mittels systematischem Problemlösen bearbeitet werden, was das Kontrollerleben stärkte. Vor dem Hintergrund der bereits erzielten Erfolge konnte die Patientin zur Exposition in sensu und damit zur emotionalen Verarbeitung ihrer überzogenen Sorgen über unlösbare Probleme (befürchteter finanzieller Abstieg der Tochter nach deren Scheidung; Sorge, den Enkelkindern könnte etwas zustoßen) motiviert werden. Infolge der Konfrontation in sensu mit zwei der als relevant identifizierten Sorgenbereiche und der gezielten Aufgabe des Vermeidungsverhaltens (Rückversicherung, Ablenkung sowie Kontrollstrategien) waren die klinisch relevante Sorgensymptomatik bei der Patientin binnen weniger Wochen remittiert sowie das Ausmaß der allgemeinen körperlichen Erregung und Anspannung deutlich reduziert.
Oft werden zum Beispiel auch Entspannungsverfahren in die Therapie integriert. Auch wenn die Effektivität multimodaler Verhaltenstherapien bei der Generalisierten Angststörung wiederholt empirisch nachgewiesen wurde [Linden et al, 2005; Übersicht: Ruhmland und Margraf, 2001], so birgt dieser Ansatz auch Nachteile: Auf der Forschungsseite lässt sich bei der Evaluation multimodaler Programme nicht erkennen, welche Programmkomponente letztlich wirksam war. Therapeutisch als problematisch einzuschätzen ist es, wenn im Rahmen einer multimodalen Strategie zwar zahlreiche unspezifische Faktoren günstig beeinflusst werden, die Schlüsselmechanismen für die Aufrechterhaltung der pathologischen Sorgen aber nur am Rande behandelt werden.

Dass eine Behandlungskonzeption, die mittels Sorgenexposition allein auf die pathologischen Sorgen abzielt, ebenso effektiv ist, konnten wir kürzlich in einer kontrollierten Studie zeigen [Hoyer et al., 2009]. Die Methode dürfte dann besonders effizient sein, wenn sowohl Therapeut als auch Patient sich darüber im Klaren sind, welche Sorgen dysfunktional und pathologisch sind, und welche nicht.

\section{Literatur}

Andor T, Gerlach A, Rist F: Superior perception of phasic physiological arousal and the detrimental consequences of the conviction to be aroused on worrying and metacognitions in GAD. J Abnorm Psychol 2008;117:193-205.

Bär T, Zubrägel D, Linden M: Kognitive Verhaltenstherapie bei generalisierten Angststörungen - das Manual der Berliner KVT-GAD-Studie. Verhaltensther Verhaltensmed 2007;28:447-462.

Becker ES, Hoyer J: Generalisierte Angststörung. Göttingen, Hogrefe, 2005.

Becker ES, Margraf J: Generalisierte Angststörung. Ein Therapieprogramm, ed 2. Weinheim, Beltz, 2007.

Becker ES, Rinck M, Margraf J, Roth WT: The emotional Stroop effect in anxiety disorders - General emotionality or disorder specificity. J Anxiety Disord 2001;15:147-159.

Becker ES, Goodwin R, Hölting C, Hoyer J, Margraf $\mathrm{J}$ : Worry and worry content in young women. J Nerv Ment Dis 2003;191:688-691.

Böhm K, Förstner U, Külz A, Voderholzer U: Versorgungsrealität der Zwangsstörungen: Werden Expositionsverfahren eingesetzt? Verhaltenstherapie 2008; 18:18-24.

Borkovec TD, Hu S: The effect of worry on cardiovascular response to phobic imagery. Behav Res Ther 1990;28:69-73.
Borkovec TD, Inz J: The nature of worry in generalised anxiety disorder: A predominance of thought activity. Behav Res Ther 1990;28:153-158.

Borkovec TD, Robinson E, Pruzinsky T, DePree JA: Preliminary exploration of worry: Some characteristics and processes. Behav Res Ther 1983;21:9-16.

Borkovec TD, Ray WJ, Stöber J: Worry: A cognitive phenomenon intimately linked to affective, physiological, and interpersonal behavioral processes. Cogn Ther Res 1998;22:561-576.

Butler G, Hope T: Manage Your Mind. The Mental Fitness Guide. Oxford, Oxford University Press, 2007.

Davey GCL: A mood-as-input account of perseverative worrying; in Davey GCL, Wells A (eds): Worry and Its Psychological Disorders. Chichester, Wiley, 2006, pp 217-238.

Davey GCL, Wells A (eds): Worry and Its Psychological Disorders. Chichester, Wiley, 2006.

Davey GCL, Tallis F, Capuzzo N: Beliefs about the consequences of worrying. Cogn Ther Res 1996;20: 499-520.

Dugas MJ, Freeston MH, Ladouceur R: Intolerance of uncertainty and problem orientation in worry. Cogn Ther Res 1997;21:593-606.

Eifert GH, Forsyth JP: Acceptance and Commitment Therapy for Anxiety Disorders. Oakland, New Harbinger, 2005.
Gerlach AL, Andor T, Patzelt J: Die Bedeutung von Unsicherheitsintoleranz für die Generalisierte Angststörung: Modellüberlegungen und Entwicklung einer deutschen Version der Unsicherheitsintoleranz-Skala. Z Klin Psychol Psychother 2008; 37:190-199.

Grawe K: Neuropsychotherapie. Göttingen, Hogrefe, 2004.

Hayes SC, Strosahl KD, Wilson KG: Acceptance and Commitment Therapy: An Experiential Approach to Behavior Change. New York, Guilford, 1999.

Helbig S, Hoyer J: Hilft wenig viel? Effekte einer Minimalintervention in der Wartezeit auf eine ambulante Psychotherapie. Verhaltenstherapie 2007;17: 109-115.

Hoyer J, Becker ES: Sorgenexposition bei Generalisierter Angststörung; in Neudeck P, Wittchen HU (Hrsg): Konfrontationstherapie bei psychischen Störungen. Göttingen, Hogrefe, 2005, pp 95-123.

Hoyer J, Beesdo K: Generalisierte Angststörung; in Wittchen HU, Hoyer J (Hrsg): Klinische Psychologie und Psychotherapie. Heidelberg, Springer, 2006.

Hoyer J, Becker ES, Roth WT: Subjective features of worry in GAD patients, social phobics and controls. Depress Anxiety 2001;13:89-96.

Hoyer J, Becker E, Margraf J: Generalized anxiety disorder and clinical worry episodes in young women. Psychol Med 2002;32:1227-1237. 
Hoyer J, Beesdo K, Becker ES: Patientenratgeber Generalisierte Angststörung. Göttingen, Hogrefe, 2007.

Hoyer J, Beesdo K, Becker ES, Wittchen HU: Epidemiologie und nosologischer Status der Generalisierten Angststörung. Z Klin Psychol Psychother 2003:32:267-275.

Hoyer J, Beesdo K, Gloster A, Höfler M, Runge J, Becker ES: Worry exposure versus applied relaxation in the treatment of generalized anxiety disorder. Psychother Psychosom 2009;78:106-115.

Linden M, Zubrägel D, Bär T, Franke U, Schlattmann P: Efficacy of cognitive behaviour therapy in generalized anxiety disorders. Results of a controlled clinical trial (Berlin CBT-GAD Study). Psychother Psychosom 2005;74:36-42.

Mennin DS, Heimberg RG, Turk CL, Fresco DM Applying an emotion regulation framework to integrative approaches to generalized anxiety disorder. Clin Psychol Sci Pract 2002;9:85-90.

Roemer L, Salters K, Raffa SD, Orsillo SM: Fear and avoidance of internal experiences in GAD: Preliminary tests of a conceptual model. Cogn Ther Res 2005;29:71-88.
Ruhmland M, Margraf J: Effektivität psychologischer Therapien von generalisierter Angststörung und sozialer Phobie: Meta-Analysen auf Störungsebene. Verhaltenstherapie 2001;11:27-40.

Ruscio AM, Borkovec TD: Experience and appraisal of worry among high worriers with and without generalized anxiety disorder. Behav Res Ther 2003; 42:1469-1482.

Ruscio AM, Borkovec TD, Ruscio J: A taxonometric investigation of the latent structure of worry. J Abnorm Psychol 2001;110:413-422.

Saß H, Wittchen HU, Zaudig M, Houben I: Diagnostisches und statistisches Manual psychischer Störungen (DSM-IV) - Textrevision - DSM-IV-TR. Göttingen, Hogrefe, 2003.

Sibrava NJ, Borkovec TD: The cognitive avoidance theory of worry; in Davey G, Wells A (eds): Worry and Its Psychological Disorders: Theory, Assessment and Treatment. Chichester, Wiley, 2006, pp 239-258.

Stiglmayr C, Stiglmayr C, Jerschke S, Schehr K: Zum Krankheitsbild der generalisierten Angststörung aus heutiger Sicht. Verhaltenstherapie 2006;16:5157.
Stöber J: Besorgnis: Ein Vergleich dreier Inventare zur Erfassung allgemeiner Sorgen. Z Diff Diagn Psychol 1995;16:50-63.

Verkuil B, Brosschot BF, Thayer JF: Capturing worry in daily life: Are trait questionnaires sufficient? Behav Res Ther 2007;45:1835-1844.

Watkins E: Constructive and unconstructive repetitive thought. Psychol Bull 2008;139:169-206.

Wells A: Cognitive Therapy of Anxiety Disorders. A Practice Manual and Conceptual Guide. Chichester, Wiley, 1997.

Wells A: A metacognitive model and therapy for Generalized Anxiety Disorder. Clin Psychol Psychother 1999;6:86-95.

Wittchen HU, Kessler RC, Beesdo K, Krause P, Höfler $\mathrm{M}$, Hoyer J: Generalized anxiety and depression in primary care: Prevalence, recognition, and management. J Clin Psychiatry 2002;63(suppl 8):24-34. 